\title{
Multimodal transport - risk and environment
}

\author{
O. Ștefanov ${ }^{*}$, AG. Agape $^{2}$, C. M. Nita ${ }^{3}$, P. Bocanete ${ }^{3}$, V. Jalan ${ }^{1}$ \\ ${ }^{1}$ Politehnica University of Bucharest, Bucharest, Romania \\ ${ }^{2}$ Head of the Underwater Research Laboratory, Diving Center, Constanta, Romania \\ ${ }^{3}$ Constanta Maritime University, 104 Mircea cel Batran Street, 900663 Constanta, Romania \\ *Coressponding author: ovidiu.stefanov2019@gmail.com
}

\begin{abstract}
The risks of multimodal transport depend on both stochastic parameters - application of the probability calculation to the results obtained by statistics, and uncertain parameters - difficult to determine. The use of a mathematical model requires a careful analysis of all the risks assigned to the multimodal transport chain, possible overload options and the consideration of the whole spectrum of control activities. The mathematical model must be able to sustain changes over time at a certain stage in order to provide options for reducing the overall risk, to capitalize on it, in particular when choosing other routes and types of transport.
\end{abstract}

\section{Introduction}

Multimodal transport can be considered as a set of separate subsystems. In this case, the subsystems are: transport types used, loading points, freight transfer points, temporary storage, and informative control of transport. From the point of view of multimodal transport, as a dynamic system for each stage of freight transport, different variants of use of separate subsystems can be considered, in particular: means of transport used, established transport routes, etc., changing them in real time. The risks of transport at each stage, for each of the subsystems may lead to an increase in the total cost, an increase in transport time, damage or loss of goods. Therefore, in order to minimize the risk in multimodal transport, it is advisable to choose the least risky means of transport. The mathematical formalization of multimodal transport begins with a description of the risks within a complex hierarchical system on several levels.

The combination of different modes of transport and the diversity of routes, specific to multimodal transport, leads to a high environmental sustainability and helps to reduce the environmental footprint of transport. The more integrated transport and logistics systems continue to be, the more obvious their impact on the environment is. In order to reduce the impact on the environment, the transport industry needs to focus on proactive environmental management. Therefore, proactive management of environmental issues refers to the interactions between transport activities that have a negative impact on the environment, the 
impact of various transport operations and facilities on the environment, alternative means of controlling and preventing environmental pollution and degradation of resources.[3] [4]

Determining the risks of multimodal transport is complicated by the fact that these risks depend not only on stochastic values, but also on uncertain values, which are difficult to predict. However, the task becomes even more complicated due to the fact that the risk probability distribution can be assessed or the risk cannot be identified. Therefore, in the risk analysis, it is necessary to operate unknown / uncertain values. Therefore, mathematical models for IT decision support for multimodal transport systems (their transhipment, selection of the most efficient routes) should be based on dynamic mathematical models. Such models should include not only possible changes in modes of transport or routes of delivery of goods, but it is also desirable to highlight the main control factors to reduce the possibility or consequences of certain problems arising from multimodal transport. [1]

Therefore, the aim of this paper is to build a mathematical model for a dynamic system of support and decision-making for multimodal transport taking into account both the risks depending on stochastic parameters and the risks caused by variables that are difficult to predict.

\section{Mathematical determination of risks in multimodal transport}

In multimodal transport, in the first stage it is possible, using the principle of decomposition, to identify those units in which the risk parameters are unknown / unpredictable, which do not require information from other parts of the transport chain to assess the risks. The principle of decomposition consists in the formal replacement of the task of identifying the transport risk of the selected transport chain option with the equivalent set of transport loads by the individual links of the specified chain. The execution of the decomposition is performed according to a certain algorithm. The first of the decomposition steps is the transformation of the selected transport route into a formalized system suitable for decomposition and the separation of the individual subsystems according to the selected characteristics. [1]

When the risk-determining parameters are represented by a universal set $\mathrm{M}$, then the set of uncertain parameters $X$ will be a set with the following form, $(m \mid \mu X(m))$ for $m \in M$. The value of the membership function $\mu \mathrm{X}$ respects the condition $\mu \mathrm{X}: \mathrm{M} \rightarrow[0,1]$, and for a separate set $(\mathrm{m} \mid \mu \mathrm{X}(\mathrm{m}))$ it shows the degree of affiliation of a certain value $\mu \mathrm{Xi}$ to the set of uncertain parameters $\mathrm{X}$. The minimum risk value, which is determined by the set of uncertain parameters $X$, will take shape: $\min U(\mathrm{~m})$, provided $\mathrm{m} \in \mathrm{M}$.

$$
\left\{m^{*}: U(m *)>U(m)\right\} \text {, provided } m \in M \text { and } m^{*} \in M
$$

Therefore, there is no single solution to the problem, but a set of such solutions. This expression formalizes the division of the system into subsystems, but does not determine the elements of the subsystems. The compression of a set $\mathrm{M}$ can be done in a tangent way, so that the overall minimum of the target function corresponds not only to the set $\mathrm{M}$, but also to the compressed set, as follows:

$$
\mathrm{Z}(\mathrm{M}) \subset \mathrm{M}
$$

Where Z (M) - compressed set.

$$
\mathrm{Z}(\mathrm{M})=\left\{\mathrm{m} *: \mathrm{U}\left(\mathrm{m}^{*}\right) \geq \mathrm{U}(\mathrm{m})\right\}, \text { provided } \mathrm{m} \in \mathrm{M}
$$


Uncertain parameter variables can be of type (L-R). The membership function $\mu X$ of such numbers is given using the functions $L(m)$ and $R(m)$. These are functions of real variables and have the following properties:

$$
\begin{gathered}
\mathrm{L}(-\mathrm{m})=\mathrm{L}(\mathrm{m}) ; \mathrm{R}(-\mathrm{m})=\mathrm{R}(\mathrm{m}) \\
\mathrm{L}(0)=\mathrm{R}(0)
\end{gathered}
$$

Where $\mathrm{m} \epsilon[\alpha, \beta]$.

$\alpha$ - represents the left limit

$\beta$ - right limit of the range of ambiguity.

Consequently, $[a, b] \epsilon[\alpha, \beta]$, where $a, b$ is the left limit and the right limit of the tolerance range, respectively. So we get:

$$
\begin{gathered}
\mu X=L[(a-m) / \alpha] \text {, for } m \in[(a-\alpha), a] \\
\mu X=R[(m-b) / \beta], \text { for } m \epsilon[b,(b+\beta)] \\
\mu X=1 \text {, for the situation where m belongs to the interval }[a, b] \\
\mu X=0 \text {, for the situation where } m \text { does not belong to the interval }[a, b]
\end{gathered}
$$

Using graph theory, multimodal transport can be described as a coherent indicative graph. An approximate graph can be represented in analytical form by the tensor equation of the following form:

$$
\mathrm{WQ}=\mathrm{Z}
$$

where the tensor W - the probability of a certain traffic flow in certain stages of the whole transport route in a given time interval, the tensor $\mathrm{Q}$ - the bandwidth of each individual section of the transport chain, the tensor $\mathrm{Z}$ - the stochastic characteristic of the possibility of passage of goods through each individual section of the transport chain. The possibility of passing goods through a separate section of the transport chain is characterized by a matrix of vectors:

$$
\vec{\Phi}:=\varphi(\mathrm{P}, \vec{\pi})
$$

Where:

$\vec{\Phi}$ - is the vector for estimating each set of event risks, it can be represented by a linear matrix; $\mathrm{P}$ - is the significance of the probability of the relevant risk factor;

$\vec{\pi}$ - the vector of the consequences of the specified risk.

The risks of multimodal transport are a dynamic system dispersed over time, which is characterized by the fact that the size of the traffic vector graph is larger than the vector of input parameters, on which depends the risk of transporting goods throughout the transport chain. After establishing the most appropriate one, from the point of view of risk minimization and mode of transport, we go through the stage of dynamic risk management, including recursive revision of the status vector of the selected variant of the specified transport route. Analyzing specialized studies on certain mathematical models for determining the risks in multimodal transport, I consider that the most convenient mathematical tool for this is the Kalman filter, because it is suitable both for finding the vector of the state of the transport graph 
and for controlling the uncertainty of the vector. Kalman's filter uses the Bayes theorem, which organically describes the dependencies between the risks of each of the stages of multimodal transport.

According to Bayes' theorem, when the probability of the transport risk is $\mathrm{P}(\mathrm{X}) \neq 0, \mathrm{P}$ $(\mathrm{Y} \mid \mathrm{X})=\mathrm{P}(\mathrm{X} \mid \mathrm{Y}) \mathrm{P}(\mathrm{Y}) / \mathrm{P}(\mathrm{X})$. In this case, the mathematical model of multimodal transport should take into account the inherent fact of multimodal freight transport for which the state of the transport process in stage $\mathrm{Y}$ depends on the state of the transport process in the previous stage $\mathrm{X}$. transport $\vec{\Delta}_{\mathrm{X}}$, for which a certain static set is characteristic of the risks and can be written in the form:

$$
\vec{\Delta}_{\mathrm{X}}=\vec{U}_{\mathrm{X}} \vec{Y}_{\mathrm{X}}+\vec{W}_{\mathrm{X}} \vec{\xi}_{\mathrm{X}}+\vec{\delta}_{\mathrm{X}}
$$

Where:

$\vec{U}_{\mathrm{X}}$ - vector of change of the transport process, inherent to the transport process in stage Y; W $\vec{W}_{\mathrm{X}}$ - the control vector, which is accompanied by a matrix (set) of control actions;

$\vec{\xi}_{\mathrm{X}}$ - the Gaussian set of transport risks, which is characterized by the risk matrix of the entire transport chain, whose diagonal is the dispersion of the components of the specified risk vector, and outside the diagonal there are co-variants of the risk components; for the risk matrix of the whole transport chain, cov $(\mathrm{m}) \geq 0$. [2]

The initial state and vectors of the individual risk components of the entire transport chain are independent values. The use of this method requires a careful analysis of all risks in the multimodal transport chain, of possible overloads and of taking into account the full spectrum of control activities. This method allows the construction of a multimodal transport process control system with the dynamic change of the individual parts of the freight transport chain, depending on the increase of the risks at certain stages or the changes of the transport conditions. Thus, it is possible in real time, by taking control of the effects on the transport process $\vec{\xi}_{\mathrm{x}}$, to reduce the risks and to obtain the minimization of the target function of the transport of goods - the cost of transport, the time or the deterioration of the goods.

The weighting of each risk or the risk of each stage of transport is the proportion of its impact on the value of the full risk, an analytical form:

$$
\mathrm{f}_{\mathrm{sum}}=\sum a i x f i
$$

Where:

$f i$ - the value of the risk or the risk of the transport stage, and - the weight of the risk. [2]

The graphical interpretation of the full risk search consisting of two components is presented in the figure below: 


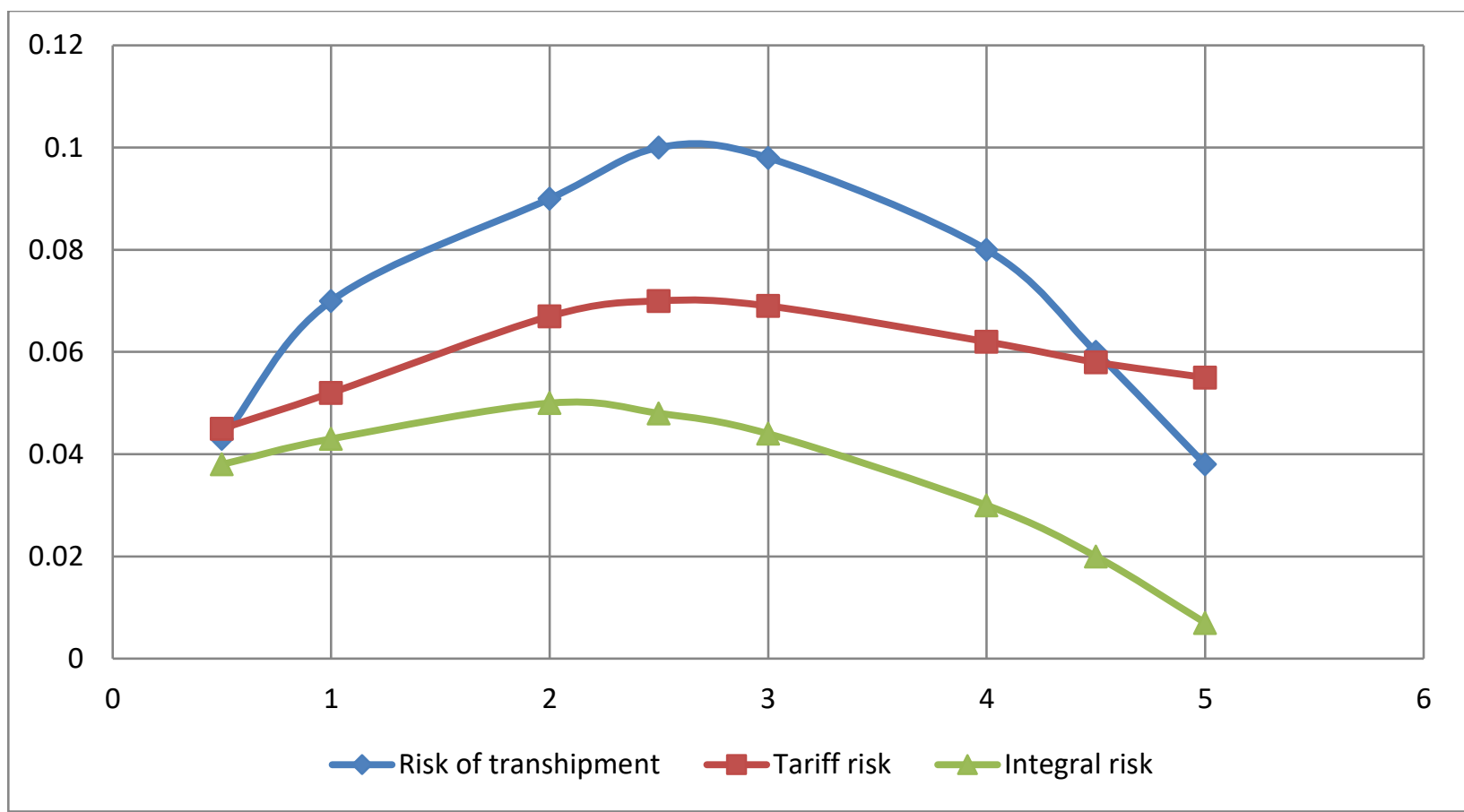

Figure 1. Graphic interpretation of the integral risk search

The analysis of finding the full risk of transport, including two risks, which have the largest share, related to the tariff and transhipment of multimodal goods (Figure 1), is characterized by the fact that the first is stochastic and the second is variable. That is, this analysis clearly demonstrates the functionality of the mathematical model, because the mathematical devices for calculating these risks are different, however, according to the proposed approach, they allow to find the full risk of multimodal transport.

\section{Conclusions}

The study showed that the risks of multimodal transport depend on stochastic quantities and statistical results, as well as variable parameters. As it is not possible to use a single mathematical device to manage such risks, a mathematical model for calculating the full risk of multimodal freight transport is suggested, which allows the use of different mathematical approaches for calculating different types of local risks in individual stages transport. The application of this mathematical model implies the need for a thorough risk analysis at each stage of the entire multimodal transport chain. This makes it possible, first of all, to determine the most convenient mode of transport, based on the minimum integral risk and the related economic consequences from the point of view of minimizing the risks. Dynamic risk management is also possible at each link of the entire transport route, by recursively reviewing the status vector of the selected version of the specified transport route.

\section{References}

[1] Vitalii Nitsenko, Sergiy Kotenko, Iryna Hanzhurenko, Abbas Mardani, Ihor Stashkevych, Maksym Karakai, "Mathematical Modeling of Multimodal Transportation Risks", Proceedings of the Fourth International Conference on Soft Computing and Data Mining (SCDM 2020), Melaka, Malaysia, January 22-23, 2020 
[2] Henrik Hult, Filip Lindskog, "Mathematical Modeling and Statistical Methods for Risk Management", Lecture Notes, 2007

[3] John A, Paraskevadakis D, Bury A, "An integrated fuzzy risk assessment for seaport operation", Safety Science, Volume 68, October 2014

[4] Jyri P., VilkoJukka P., Hallikas M., "Risk assessment in multimodal supply chains", International Journal of Production Economics, Volume 140, Issue 2, December 2012

[5] Novac V., Rusu E., Scurtu I. C., Opportunities and Risks Related to Offshore Activities in the Western Black Sea, Journal of Environmental Protection and Ecology 20, No 4, 16981707 (2019)

[6] Sum, C., Lau, Y. Y., \& Dragomir, C. (2020). Responsible Ferry, Happy Employees, and Quality Services: The Study of Fair Transport in the Ferry Industry: The Study of Fair

Transport in the Ferry Industry. Technium: Romanian Journal of Applied Sciences and

Technology, 2(4), 1-5. https://doi.org/10.47577/technium.v2i4.840

[7] Mateescu, C., Dima, A., \& Marin, D. (2020). Sustainable solution for alien algae management to reduce the environmental consequences of sea and river transport. Technium:

Romanian Journal of Applied Sciences and Technology, 2(1), 97-103.

https://doi.org/10.47577/technium.v2i1.67

[8] Lupchian, M. (2020). Influence of propulsion installation performance on travel efficiency. Technium: Romanian Journal of Applied Sciences and Technology, 2(7), 50-53. https://doi.org/10.47577/technium.v2i7.1644 